\title{
Atividade antifúngica de Caryocar brasiliensis (Caryocaraceae) sobre Cryptococcus neoformans
}

\author{
Antifungal activity of Caryocar brasiliensis (Caryocaraceae) \\ against Cryptococcus neoformans
}

\begin{abstract}
Xisto Sena Passos ${ }^{1}$, Suzana da Costa Santos ${ }^{2}$, Pedro Henrique Ferri², Orionalda de Fátima Lisboa Fernandes ${ }^{1}$,Thaliana de Freitas Paula ${ }^{1}$, Ana Cristina Ferreira Garcia ${ }^{1}$ e Maria do Rosário Rodrigues Silva ${ }^{1}$
\end{abstract}

\begin{abstract}
Resumo A grande incidência de criptococose em decorrência do aumento crescente de indivíduos imunodeprimidos e os efeitos colaterais aos fármacos utilizados para o tratamento desta infecção, tem incentivado a pesquisa de novos agentes antifúngicos. Através da técnica de diluição em ágar, foi verificada a atividade antifúngica (in vitro) de diferentes constituintes de Caryocar brasiliensis sobre Cryptococcus neoformans. Verificou-se que a cera epicuticular retirada da folha, coletada em período de baixo índice pluviométrico (170,8mm de água), foi a parte mais ativa da planta, inibindo o crescimento de 91,3\% (21/23) dos isolados de Cryptococcus neoformans em concentração $\leq a 250 \mu \mathrm{g} / \mathrm{mL}$.
\end{abstract}

Palavras-chaves: Caryocar brasiliensis. Planta do Cerrado. Cryptococcus neoformans. Suscetibilidade in vitro.

Abstract The widespread occurrence of cryptococcosis mainly in immunocompromised patients and the side effects of available drugs which are effective against this mycosis have led investigators to search for new antimycotic agents. Caryocar brasiliensis derived compounds were investigated against Cryptococcus neoformans using the agar dilution method. Based on MIC values, the best results were obtained with a concentration of $\leq 250 \mu \mathrm{g} / \mathrm{mL}$ of cuticular waxes of the Caryocar brasiliensis leaf collected during the dry period (170.8mm of precipitation) which inhibited the growth of $91.3 \%$ (21/23) Cryptococcus neoformans isolates.

Key-words: Caryocar brasiliensis. Cerrado plant. Cryptococcus neoformans. Antifungal activity.

Nas últimas décadas, tem sido observado um aumento acentuado de infecções fúngicas, as quais contribuem para uma elevada taxa de mortalidade em pacientes imunocomprometidos ${ }^{5}{ }^{16}$. A criptococose causada por Cryptococcus neoformans é considerada micose oportunista, freqüentemente diagnosticada produzindo lesões principalmente no sistema nervoso central em pacientes com AIDS ${ }^{91123}$

O tratamento da meningite criptocócica na fase aguda para pacientes imunocomprometidos bem como para imunocompetentes é feito normalmente utilizandose anfotericina B ou anfotericina B associada a 5- fluorcitosina. Para pacientes infectados pelo HIV utilizase inicialmente anfotericina B com ou sem 5-fluorcitosina seguido por uma terapia de manutenção com fluconazol ${ }^{18}$. Os efeitos colaterais verificados com estes antifúngicos fazem com que se busque alternativas ao tratamento desta micose $\mathrm{f}^{1022}$. Compostos com características antifúngica extraídos da vegetação nativa, que são encontrados em abundância no território brasileiro, poderiam constituir uma alternativa terapêutica ${ }^{2}{ }^{4}$. Caryocar brasiliensis Camb (Caryocaraceae), que produz o fruto conhecido como pequi e distribuído amplamente nas regiões central e sudeste do $\mathrm{Brasil}^{3}$ tem sido relatado

1. Instituto de PatologiaTropical e Saúde Pública da Universidade Federal de Goiás, Goiânia, GO. 2. Instituto de Química da Universidade Federal de Goiás, Goiânia, GO.

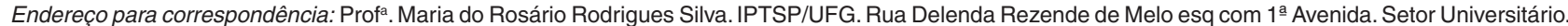
Caixa Postal 131, 74605-050, Goiânia, GO

Fone: 5562 209-6127

e-mail rosario@iptsp.ufg.br.

Recebido para publicação em 15/1/2002 
por possuir atividade antifúngica. $\mathrm{O}$ óleo essencial das sementes desta planta mostra bioatividade em testes de suscetibilidade in vitro sobre isolados de $C$. neoformans e Paracoccidioides brasiliensis ${ }^{14}$.

Neste trabalho, foi descrita a atividade antifúngica das folhas (extrato bruto etanólico, fração acetato de etila e cera epicuticular), dos dois principais componentes presentes no óleo essencial das sementes, além dos óleos fixos da amêndoa e da semente de $C$. brasiliensis sobre isolados de $C$. neoformans var. neoformans e de $C$. neoformans var gattii.

\section{MATERIAL E MÉTODOS}

Material botânico. Espécimes de $C$. brasiliensis Camb foram coletadas de diferentes regiões do Brasil e períodos do ano. Os frutos maduros foram provenientes das cidades de São Gonçalo do Abaeté (Minas Gerais) e de Goiânia (Goiás), coletados no mês de dezembro de 1997 e dezembro de 1998, nas cidades de Paraúna e Goiânia (Goiás) em agosto de 1997 e em dezembro de 1998, respectivamente, enquanto a cera epicuticular das folhas foi obtida de 56' 17"; 769m de altitude) nos meses de alta pluviosidade (março; 207,9mm de água) e baixa pluviosidade (outubro; $170,8 \mathrm{~mm}$ de água). Todos os espécimes utilizados foram depositados no Herbarium da Universidade Federal de Goiás (UFG), Brasil.

Extratos da planta. As folhas de C. brasiliensis $(0,35 \mathrm{~kg})$ foram secas em estufa com ventilação forçada $\left(40^{\circ} \mathrm{C}\right)$, moídas em moinhos de facas até atingirem uma baixa granulometria e em seguida sofreram percolação a frio em etanol $96 \%$ ( $3 \times 500 \mathrm{~mL})$, ao abrigo da luz e sob agitação durante 24 horas. Filtração do sobrenadante e evaporação do solvente à pressão reduzida conduziu ao extrato bruto etanólico, EBE $(19,6 \mathrm{~g} ; 5,6 \%$ de rendimento). Parte do EBE (10g) foi água destilada $(1: 1 \mathrm{v} / \mathrm{v})$. Separação da fase orgânica, conduziu à fase aquosa livre de graxas e clorofilas. Posterior extração da fase aquosa com acetato de etila (3 x 100mL) e evaporação do solvente orgânico, conduziu à fração acetato de etila $(0,36 \mathrm{~g})$. Para a obtenção da cera epicuticular, folhas verdes recémcoletadas $(25 \mathrm{~g})$ foram percoladas em éter etílico $(50 \mathrm{~mL})$ durante 30 segundos. Após evaporação do solvente sob pressão reduzida, obteve-se apenas a cera da superfície epicuticular com rendimentos de 0,36\% (89mg) e 0,39\% (97,5mg), para os meses de março e outubro, respectivamente.

Os óleos fixos da semente e da amêndoa foram adquiridos comercialmente em Goiânia (Mercado Central), em fevereiro de 2000 , enquanto os ésteres hexanoato de etila e octanoato de etila, foram adquiridos da Sigma Chemical Co (USA). respectivamente. As folhas desta planta foram coletadas espécimes coletados em Goiânia (S 16운'24"/ W 48을 submetido à partição líquido-líquido com éter etílico-

Microrganismos. Foram utilizados 23 isolados de C. neoformans, sendo 19 C. neoformans var. neoformans e 4 C. neoformans var. gattii, devidamente identificados no laboratório de Micologia do Instituto de Patologia Tropical e Saúde Pública da UFG. Todos os isolados foram obtidos de indivíduos portadores do vírus da imunodeficiência humana (HIV), acometidos de meningite criptocócica, provenientes do Hospital de Doenças Tropicais do Estado de Goiás, Brasil. C. neoformans var. neoformans (CBS 132), foi usada como padrão. Todos os fungos estudados foram mantidos em ágar Sabouraud Dextrose à temperatura ambiente (BBL, Beckton Dickinson Microbiology Systems, USA), para posterior avaliação de suscetibilidade.

Teste de suscetibilidade antifúngica. A atividade antifúngica foi realizada segundo a técnica de diluição em ágar, proposta por Alves \& Cury ${ }^{1}$, modificada. Extrato bruto etanólico e sua fração acetato de etila, os óleos fixos da semente e da amêndoa, a cera da superfície epicuticular e os dois principais componentes dos óleos essenciais (hexanoato de etila e octanoato de etila) de $C$. brasiliensis $(100 \mathrm{mg})$ foram solubilizados em $1 \mathrm{~mL}$ de dimetilsulfóxido (DMSO), diluídos ao dobro em caldo Yeast Nitrogen Base (YNB) (DIFCO) e, posteriormente distribuídos em placas de Petri contendo $20 \mathrm{~mL}$ de ágar YNB de maneira que se obtivesse uma concentração que variasse de $1.000 \mu \mathrm{g} / \mathrm{mL}$ a $15,6 \mu \mathrm{g} / \mathrm{mL}$. Três microlitros da suspensão das células preparadas em solução fisiológica estéril, homogeneizadas e ajustadas com espectrofotômetro em comprimento de onda de $530 \mathrm{~nm}$, de forma a obter $10^{6}$ células $/ \mathrm{mL}$ foram semeados com o auxílio de uma micropipetadora sobre o ágar em pontos eqüidistantes nas placas de Petri, contendo ágar YNB nas diferentes concentrações dos extratos. A concentração inibitória mínima (CIM) obtida após incubação a $37^{\circ} \mathrm{C}$ por 72 horas foi definida como a menor concentração da amostra teste capaz de inibir completamente o crescimento visível do fungo. Paralelamente, utilizando-se a mesma técnica efetuou-se o teste de suscetibilidade de todos os isolados de C. neoformans, frente ao fluconazol (Pfizer). O fluconazol foi dissolvido em $1 \mathrm{~mL}$ de água destilada, diluído ao dobro em caldo YNB e distribuído em $20 \mathrm{~mL}$ de ágar YNB de tal modo que as concentrações variaram de $15,6 \mu \mathrm{g} / \mathrm{mL}$ a $0,03 \mu \mathrm{g} / \mathrm{mL}$.

\section{RESULTADOS}

O extrato bruto etanólico das folhas de $C$. brasiliensis mostrou-se com atividade antifúngica sobre $C$. neoformans var. neoformans, sendo que $89,5 \%$ dos isolados foram inibidos em uma concentração menor ou igual a $1.000 \mu \mathrm{g} / \mathrm{mL}$. A cera da superfície epicuticular das folhas, coletada no período de baixo índice pluviométrico (outubro), mostrou-se com maior atividade biológica do que a cera proveniente do mês com elevado 
índice pluviométrico (março). A cera epicuticular das folhas de outubro inibiu o crescimento de 73,7\% (14/19) dos isolados de $C$. neoformans var neoformans à uma concentração menor do que $250 \mu \mathrm{g} / \mathrm{mL}$, sendo que $42,1 \%$ (8/19) ocorreu à uma concentração de $15,6 \mu \mathrm{g} / \mathrm{mL}$.

A bioatividade dos óleos fixos da semente e da amêndoa de $C$. brasiliensis mostrou-se bastante elevada para $C$. neoformans. Todos os isolados foram inibidos por estes óleos a uma concentração de $1.000 \mu \mathrm{g} / \mathrm{mL}$, sendo que o óleo fixo da semente, o mais ativo, inibiu $21,1 \%$ dos isolados em uma concentração de $15,6 \mu \mathrm{g} /$ $\mathrm{mL}$, enquanto o óleo fixo da amêndoa foi capaz de inibir $10,5 \%$ em uma concentração de $62,5 \mu \mathrm{g} / \mathrm{mL}$.

Os resultados da ação antifúngica da fração acetato de etila, obtida a partir do extrato bruto etanólico das folhas e dos dois componentes majoritários presentes nos óleos essenciais dos frutos de $C$. brasiliensis demonstraram que a fração acetato de etila possui elevada atividade antifúngica, apresentando CIM de
$31,3 \mu \mathrm{g} / \mathrm{mL}$ para $4(21 \%)$ isolados e de $62,5 \mu \mathrm{g} / \mathrm{mL}$ para $8(42,1 \%)$, enquanto o hexanoato de etila apresentou o mais baixo potencial antifúngico, sendo capaz de inibir apenas $4(15,8 \%)$ isolados de $C$. neoformans na concentração de $500 \mu \mathrm{g} / \mu \mathrm{L}$.

A análise de suscetibilidade in vitro com relação às duas variedades mostrou que $C$. neoformans var gattii apresentou-se com menor sensibilidade aos extratos de C. brasiliensis do que C. neoformans var neoformans. Os resultados de CIM do extratos bruto etanólico, das ceras, dos óleos fixos, da fração acetato de etila, de octanoato de etila e de hexanoato de etila para os isolados de C. neoformans var. neoformans e C. neoformans var gattii estão apresentados na Tabela 1.

A suscetibilidade dos isolados de $C$. neoformans ao agente antifúngico fluconazol apresentou valores de CIM variando de $15,6 \mu \mathrm{g} / \mathrm{mL}$ a $1,95 \mu \mathrm{g} / \mathrm{mL}$. Verificou-se que 9 isolados de $C$. neoformans mostraram-se com CIM igual a 15,6 $\mu \mathrm{g} / \mathrm{mL}$ (Tabela 2 ).

Tabela 1 - Atividade antifúngica de C. brasiliensis sobre 23 isolados de C. neoformans.

\begin{tabular}{|c|c|c|c|c|c|c|c|c|c|}
\hline \multirow[b]{2}{*}{ C. brasiliensis } & \multirow[b]{2}{*}{ C. neoformans } & \multicolumn{8}{|c|}{$\begin{array}{l}\text { Porcentagem acumulativa de inibição de } C \text {. brasiliensis em diferentes } \\
\text { concentrações }(\mu \mathrm{g} / \mathrm{mL}) \text { sobre isolados de } C \text {. neoformans. }\end{array}$} \\
\hline & & 15,6 & 31,3 & 62,5 & 125 & 250 & 500 & 1000 & $>1000$ \\
\hline \multicolumn{10}{|c|}{ Extratto bruto etanólico } \\
\hline & var neoformans ${ }^{1}$ & 0 & 0 & 0 & 0 & 42,1 & 63,2 & 89,5 & 100,0 \\
\hline & var gattii ${ }^{2}$ & 0 & 0 & 0 & 0 & 0 & 0 & 100,0 & 100,0 \\
\hline & CBS $132^{3}$ & 0 & 0 & 0 & 0 & 0 & 100,0 & 100,0 & 100,0 \\
\hline \multicolumn{10}{|c|}{ Cera epicuticular (março) } \\
\hline & var neoformans & 0 & 0 & 0 & 0 & 0 & 10,5 & 10,5 & 100,0 \\
\hline & var gattii & 0 & 0 & 0 & 0 & 0 & 0 & 0 & 100,0 \\
\hline & CBS 132 & 0 & 0 & 0 & 0 & 0 & 0 & 100,0 & 100,0 \\
\hline \multicolumn{10}{|c|}{ Cera epicuticular (outubro) } \\
\hline & var neoformans & 42,1 & 47,4 & 57,9 & 73,7 & 100,0 & 100,0 & 100,0 & 100,0 \\
\hline & var gattii & 0 & 0 & 0 & 0 & 0 & 0 & 100,0 & 100,0 \\
\hline & CBS 132 & 0 & 0 & 0 & 0 & 0 & 100,0 & 100,0 & 100,0 \\
\hline \multicolumn{10}{|c|}{ Óleo fixo da semente } \\
\hline & var neoformans & 21,1 & 21,1 & 21,1 & 21,1 & 36,9 & 73,7 & 100,0 & 100,0 \\
\hline & var gattii & 0 & 0 & 0 & 0 & 0 & 0 & 100,0 & 100,0 \\
\hline & CBS 132 & 0 & 0 & 0 & 0 & 0 & 0 & 100,0 & 100,0 \\
\hline \multicolumn{10}{|c|}{ Óleo fixo da amêndoa } \\
\hline & var neoformans & 0 & 0 & 10,5 & 10,5 & 21,0 & 57,8 & 100,0 & 100,0 \\
\hline & var gattii & 0 & 0 & 0 & 0 & 0 & 0 & 100,0 & 100,0 \\
\hline & CBS 132 & 0 & 0 & 0 & 0 & 100,0 & 100,0 & 100,0 & 100,0 \\
\hline \multicolumn{10}{|c|}{ Fração acetato de etila } \\
\hline & var neoformans & 0 & 21,0 & 63,1 & 78,9 & 84,2 & 84,2 & 89,5 & 100,0 \\
\hline & var gattii & 0 & 0 & 0 & 0 & 0 & 25,0 & 50,0 & 100,0 \\
\hline & CBS 132 & 0 & 0 & 0 & 100,0 & 100,0 & 100,0 & 100,0 & 100,0 \\
\hline \multicolumn{10}{|c|}{ Octanoato de etila } \\
\hline & var neoformans & 0 & 0 & 0 & 0 & 0 & 0 & 52,6 & 100,0 \\
\hline & var gattii & 0 & 0 & 0 & 0 & 0 & 0 & 50,0 & 100,0 \\
\hline & CBS 132 & 0 & 0 & 0 & 0 & 0 & 0 & 100,0 & 100,0 \\
\hline \multicolumn{10}{|c|}{ Hexanoato de etila } \\
\hline & var neoformans & 0 & 0 & 0 & 0 & 5,3 & 15,8 & 21,1 & 100,0 \\
\hline & var gattii & 0 & 0 & 0 & 0 & 0 & 0 & 0 & 100,0 \\
\hline & CBS 132 & 0 & 0 & 0 & 0 & 0 & 0 & 100,0 & 100,0 \\
\hline
\end{tabular}


Tabela 2 - Atividade antifúngica de fluconazol sobre 23 isolados de C. neoformans.

\begin{tabular}{|c|c|c|c|c|}
\hline \multirow[b]{2}{*}{ C. neoformans } & \multicolumn{4}{|c|}{$\begin{array}{l}\text { Porcentagem acumulativa de inibição de fluconazol em diferentes } \\
\text { concentrações }(\mu \mathrm{g} / \mathrm{mL}) \text { sobre isolados de } C \text {. neoformans }\end{array}$} \\
\hline & 1,95 & 3,9 & 7,8 & 15,6 \\
\hline var neoformans $^{1}$ & 5,3 & 31,6 & 63,2 & 100,0 \\
\hline var gattii ${ }^{2}$ & 0 & 25,0 & 50,0 & 100,0 \\
\hline CBS $132^{3}$ & 0 & 0 & 100,0 & 100,0 \\
\hline
\end{tabular}

\section{DISCUSSÃO}

Os resultados in vitro mostraram que $C$. brasiliensis apresenta potencial atividade antifúngica sobre os isolados de C. neoformans. Dos extratos, frações e compostos avaliados, verificou-se maior atividade antifúngica com a cera da superfície epicuticular coletada no mês de outubro, e da fração acetato de etila do extrato bruto etanólico das folhas. As diferenças quantitativas em relação às concentrações inibitórias observadas para C. brasiliensis podem ser justificadas pelos constituintes químicos presentes nos diferentes materiais botânicos analisados. A maior atividade antifúngica da cera epicuticular das folhas coletada no mês de outubro, quando comparada com a proveniente da coletada no mês de março (Tabela 1) sugere um mecanismo de defesa química da planta, principalmente em períodos de menor precipitação de água e maior predação por insetos. De fato, hidrocarbonetos de cadeia longa acumulados na cera foliar, atuam como os responsáveis pela dissuasão do comportamento forrageador de saúvas ${ }^{20}$, envolvendo-se também na resistência da planta a ataques de microrganismos ${ }^{17}$. Por outro lado, a maior atividade antifúngica do óleo fixo da semente comparado ao óleo fixo da amêndoa (Tabela 1), pode ser atribuída a composição química da primeira, constituída por ácidos graxos de natureza saturada, ausentes na amêndoa ${ }^{3}$. Já a resposta biológica apresentada pela fração acetato de etila do extrato bruto etanólico (Tabela 1) provavelmente encontra-se relacionada a sua composição química majoritariamente composta de taninos condensados (monômeros, dímeros e trímeros), flavonóides glicosilados e taninos hidrolisáveis, responsáveis pela ação antifúngica dessa mesma fração frente a outros fungos, como P. brasiliensis ${ }^{8}$.

Atividade biológica de outras plantas de uso na medicina popular tem sido observada para
C. neoformans. O óleo de Piper angustifolium apresentou CIM de $50 \mu \mathrm{g} / \mathrm{mL}$ para um isolado de $C$. neoformans ${ }^{21}$. Trabalho realizado com berberina, o principal componente alcaloídico de Xanthorhiza simplicissima mostrou que este composto possui excelente atividade sobre $C$. neoformans, apresentando uma CIM de $1,56 \mu \mathrm{g} / \mathrm{mL}$ sobre este microrganismo ${ }^{12}$. O óleo da folha de Eugenia dysenterica mostrou potente ação inibitória sobre este fungo, havendo uma inibição em uma concentração de $250 \mu \mathrm{g} / \mathrm{mL}^{7}$.

Os isolados de C. neoformans var. gattii utilizados neste trabalho apresentaram valores de CIM mais elevados do que os observados para $C$. neoformans var. neoformans para todos os extratos, frações e compostos avaliados. C. neoformans var gattii tem sido relatada principalmente, como causa de doença em pacientes não imunocomprometidos ${ }^{131519}$.

A análise comparativa entre fluconazol e a cera epicuticular coletada no mês de outubro que mostrouse a mais ativa, não forneceu resultados similares. A cera inibiu 8 isolados na concentração de $15,6 \mu \mathrm{g} / \mathrm{mL}$ enquanto que o fluconazol foi capaz de inibir todos os isolados de $C$. neoformans nesta mesma concentração. Diante destes resultados verificou-se que a atividade antifúngica expressa pelo fluconazol foi superior a cera, no entanto, fluconazol apresentou CIM de $15,6 \mu \mathrm{g} / \mathrm{mL}$ para $9(13 \%)$ isolados (Tabela 2$)$.

A grande incidência de infecções, principalmente em indivíduos imunocomprometidos, aumenta a importância da procura e descoberta de compostos terapêuticos alternativos. A atividade antifúngica de C. brasiliensis para $C$. neoformans observada neste trabalho, pode abrir perspectivas no sentido de desenvolver um fitoterápico eficaz e de baixo custo.

\section{AGRADECIMENTOS}

Os autores agradecem ao CNPq/PCOP, FUNAPE/UFG pelo suporte financeiro e ao CNPq/PIBIC/UFG pelos bolsistas A.C.F Garcia e T.F. Paula.

\section{REFERÊNCIAS BIBLIOGRÁFICAS}

1. Alves SH, Cury AE. Sensibilidade de leveduras do gênero Candida isoladas de pacientes com câncer, a antifúngicos poliênicos. Revista do Instituto de Medicina Tropical de São Paulo 34:251-254, 1992.
2. Alves TMA, Silva AF, Brandão M, Grandi TS, Smânia EF, Smânia Jr A, Zani CL. Biological screening of Brazilian medicinal plants. Memórias do Instituto Oswaldo Cruz 95:367-373, 2000. 
3. Araujo FD. A review of Caryocar brasiliensis (Caryocaraceae) An economically valuable species of the Central Brazilian Cerrados. Economy Botany 49: 40-48, 1995.

4. Cabrera AL, Klein RM. Compostas. Tribo: Vernoniae. In: Reitz. Revista Flora llustrada Catarinense. Itajai-SC. Imprensa Oficial do Estado de Santa Catarina 3: 224-408, 1980.

5. Canton E, Viudes A, Pemán. J. Infección sistémica nosocomial por levaduras. Revista Iberoamericana de Micología 18:51-55, 2001.

6. Carrillo-Muñoz AJ, Brió S, Quindós G. Una nueva generación de fármacos antifúngicos. Revista Iberoamericana de Micología 18:2-5, 2001.

7. Costa TR, Fernandes OFL, Santos SC, Oliveira CMA, Lião LM, Ferri PH, Paula JR, Ferreira HD, Sales BHN, Silva MRR. Antifungal activity of volatile constituents of Eugenia dysenterica leaf oil. Journal of Ethnopharmacology 72:111-117, 2000.

8. Guimarães DO, Ribeiro JP, Santos SC, Ferri PH, Garcia ACF, Pires JS, Castro ACM, Silva MRR, Ferreira HD. Atividade antifúngica de taninos de Caryocar brasiliensis Camb. In: Resumos da V Jornada Paulista de Plantas Medicinais, Botucatu, p. 158, 2001.

9. Gumbo T, Hakim JG, Mielke J, Siwji S, Just-Nubling G, Ismail A. Cryptococcus myelitis: atypical presentation of a common infection. Clinical Infectious Diseases 32:1235-1236, 2001.

10. Kulberg BJ. Trends in immunotherapy of fungal infections. European Journal of Clinical Microbiology Infectious Diseases 16:51-55, 1997.

11. Negroni R, Arechavala A, Robles AM, Bianchi M, Bava A, Helou S. Revision clínica y evolución terapéutica de pacientes con criptococosis asociada al sida. Revista Iberoamericana de Micología 12:12-15, 1995.

12. Okunade AL, Hufford CD, Richardson MD, Peterson JR, Clark AM. Antimicrobial properties of alkaloids from Xanthorhiza simplicissima. Journal of Pharmaceutical Sciences. 83:404-406, 1994.

13. Passoni LFC. Wood, animals and human beings as reservoirs for human Cryptococcus neoformans infectious. Revista Iberoamericana de Micología 16:77-81,1999.
14. Passos XS, Costa M, Souza LKH, Miranda ATB, Lemos AA, Ferri $\mathrm{PH}$, Santos SC, Silva MRR. Antifungal activity of Caryocar brasiliensis against Paracoccidioides brasiliensis and Histoplasma capsulatum. Anais do XXI Congresso Brasilieiro de Microbiologia 60:71, 2001.

15. Rozenbaum R, Gonçalves AJR, Wanke B, Vieira W. Cryptococcus neoformans var. gattii in a brasilian AIDS patient. Mycopathologia 112:33-34, 1990.

16. Rubin RH. Infectious disease complications of renal transplantation. Kidney International 44:221-236, 1993.

17. Schoonhoven LM, Jeremy T, Van Loon JJA. Insect-Plant Biology from Physiology to Evolution. Chapman \& Hall, London, 1998.

18. Sheelan DJ, Hitchcock CA, Sibley CM. Current and emerging azole antifungal agents. Clinical Microbiology Reviews 12:40$79,1999$.

19. Speed B, Dunt D. Clinical and host differences between infections with the two varieties of Cryptococcus neoformans. Clinical Infectious Diseases 21:28-34, 1995.

20. Sugayama RL, Salatino A. Influence of leaf epicuticular waxes from cerrado species on substrate selection by Atta sexden rubropilosa. Entomologia Experimentalis et Applicata 74: 63-69, 1995.

21. Tirillini B, Velasquez ER, Pellegrino R. Chemical composition and antimicrobial activity of essential oil of Piper angustifolium. Planta Medica 62:372-373. 1996.

22. Vanden-Bossche H. Mechanisms of antifungal resistance. Revista Iberoamericana de Micología 14:44-49, 1997.

23. Vander-der Host CM, Saag CM, Cloud GA, Hamill RJ, Graybill J, Sobel JD, Johnson PC, Tuazon CV, Kerkering T, Moskovitz BL, Powderly WG, Dismukes WE. National Institute of Allergy and Infectious Diseases Mycosis Study Group AIDS Clinical Trials Group. Treatment of cryptococcal meningitis associated with the acquired immunodeficiency syndrome. New England Journal of Medical 337:15-21, 1997. 